Revue des patrimoines

19 | 2012

Patrimoines et conservation préventive. Pratiques comparées et nouveaux enjeux

\title{
Une formation interactive sur trois ans. La conservation préventive, un travail d'équipe
}

Marie-Dominique Parchas

\section{(2) OpenEdition}

Journals

Édition électronique

URL : http://journals.openedition.org/insitu/9837

DOI : 10.4000/insitu.9837

ISSN : 1630-7305

Éditeur

Ministère de la culture

\section{Référence électronique}

Marie-Dominique Parchas, « Une formation interactive sur trois ans. La conservation préventive, un travail d'équipe », In Situ [En ligne], 19 | 2012, mis en ligne le 25 septembre 2012, consulté le 20 avril 2019. URL : http://journals.openedition.org/insitu/9837 ; DOI : 10.4000/insitu.9837

Ce document a été généré automatiquement le 20 avril 2019

\section{cc) (1)}

In Situ Revues des patrimoines est mis à disposition selon les termes de la licence Creative Commons Attribution - Pas d'Utilisation Commerciale - Pas de Modification 4.0 International. 


\title{
Une formation interactive sur trois ans. La conservation préventive, un travail d'équipe
}

\author{
Marie-Dominique Parchas
}

1 La direction des Archives de France propose depuis 2008 une formation sur site originale par sa conception inspirée du projet «Teamwork in preventive conservation » de l'Institut international de Conservation, l'ICCROM. Elle s'intitule « la conservation préventive, un travail d'équipe. Suivi sur trois années ». Elle allie sensibilisation à la conservation préventive de l'ensemble ou d'une partie du personnel et management de projets au plus près des préoccupations de chaque service. Cette formation est interactive, les services qui postulent doivent avoir des projets et libérer du temps pour les réaliser. Une sélection a été faite en fonction de ces projets. La fréquence des déplacements, l'implication de l'animateur ne permettent pas de retenir un nombre important de services. Depuis 2008, 12 services ont participé à ce programme : archives départementales ou municipales mais aussi un musée, ce qui représente un total de 168 agents formés.

Il est conseillé d'associer les responsables des bâtiments et des installations de climatisation, le SDIS (pompiers) de manière à avoir une vision globale des actions de conservation préventive, du bâtiment aux collections avec gestion des risques.

Des outils spécifiques ont été mis en place pour évaluer les besoins mais aussi les actions en se basant sur la Méthodologie d'évaluation des pratiques en conservation préventive, élaborée par le Centre Interrégional de Conservation du Livre à la demande des Archives de France en 2008. Un résumé sur tableur Excel avec une représentation graphique permet d'en faire une synthèse aisée. Le tout est accessible sur le site des Archives de France.

4 La première session se déroule en général sur trois jours, les suivantes sur une ou deux journées consacrées tous les six mois au bilan des actions des groupes de travail et si besoin à des formations complémentaires. 
5 Les trois jours consacrés à la sensibilisation à la conservation préventive incluent une visite critique des locaux et un brain storming. Celui-ci a pour objectifs de repérer les problèmes liés à la conservation préventive et de constituer des groupes de travail : bilan des pratiques, rédaction de protocoles de manière à ce que l'ensemble du personnel ait des références écrites et consensuelles sur les actions à mener comme par exemple sur le protocole d'introduction de nouveaux versements, la communication en salle de lecture, le suivi du climat, les plans d'urgence... Les groupes doivent se réunir si possible au moins une fois par mois, rédiger un compte-rendu et d'éventuelles questions qui sont transmis à l'animatrice de la formation, Marie-Dominique Parchas.

Des formations complémentaires peuvent être données par d'autres intervenants en fonction de la demande: manipulation, dépoussiérage et conditionnement, ateliers pratiques animés par des techniciens d'art du département de la conservation des Archives nationales ou sur d'autres sujets comme constat d'état, plan d'urgence, prise en charge des fonds photographiques et audiovisuels, grands formats....

7 Le département de la formation scientifique et technique de la direction générale des patrimoines prend en charge l'ensemble des frais. L'animatrice rédige le compte-rendu des trois jours qui inclue un audit de situation et les objectifs à atteindre. A l'issue des trois années, un bilan général est effectué par les services. Il est plus ou moins riche en fonction de leur implication.

8 Les avantages de cette formation sont conséquents : vérification au cours des trois années des connaissances acquises, adaptation aux besoins, incitation ou consolidation des relations avec des services du Conseil général, avec le SDIS, des services proches (AD, AM, musées), mise en place de protocoles écrits et d'un véritable travail d'équipe, nomination de responsables, évaluation des résultats...

9 Il n'y a pas d'inconvénients mais des difficultés :

- un manque d'habitude de travail en équipe, les problèmes relationnels en interne peuvent générer une difficulté de travail en groupes. Il peut alors être plus facile de choisir un petit groupe représentatif des corps de métiers plutôt que l'ensemble du personnel ;

- l'autre difficulté peut venir du manque de temps disponible ou du manque de personnel. Les actions nécessaires à l'amélioration des conditions de conservation préventive peuvent alors être confiées à des prestataires externes ;

- trois années de collaboration peuvent paraître longues : essoufflement des groupes, manque de temps ou autonomie acquise, le train est en vitesse de croisière. Il est alors souhaitable de raccourcir cette collaboration ou d'effectuer des bilans annuels et non semestriels ;

- enfin, le travail du coordinateur est très conséquent.

10 Deux services ont préféré d'un commun accord avec l'animatrice interrompre la formation tout en restant en relation pour régler des questions ponctuelles de conservation.

11 Cette formation in situ avec managements de groupes de travail est exportable dans tout autre service patrimonial ainsi que les outils d'évaluation des pratiques en conservation préventive avec quelques ajustements nécessaires. La capacité d'écoute et d'adaptation aux besoins est la clé de sa réussite. 


\section{RÉSUMÉS}

Présentation d'une formation sur la conservation préventive in situ dans des services d'archives et un musée ayant pour originalité la mise en place de groupes de travail, leur suivi sur trois ans et l'évaluation des pratiques en conservation préventive actualisée régulièrement.

Overview of training on preventive conservation in situ in archives and museum with the original setting up of working groups, their three years follow-up and evaluation of preventive conservation practices regularly updated.

\section{INDEX}

Mots-clés : formation, conservation préventive, évaluation, management de groupes de travail.

\section{AUTEUR}

\section{MARIE-DOMINIQUE PARCHAS}

Chargée de mission pour les questions de conservation, Archives de France mariedominique.parchas@culture.gouv.fr 